\title{
Structural morphology and self-organization
}

\author{
E. Stach \\ University of Tennessee College of Architecture \& Design, USA
}

\begin{abstract}
This paper categorizes self-organization processes in nature into four categories: physics, mathematics, statics, and mechanics - abstracting these processes into simplified analytical methods. It is also an investigation into some fundamental principles concerning the logic of form optimization in nature in relation to special and physical constraints. One of the chief aims of this study is to make a contribution towards a true and complete understanding of optimization processes in nature by dealing with these processes according to their origins, principles, processes, essential features and scope. It has been attempted to establish, both by argument and evidence, that the same optimization processes used in nature can be used in architecture.
\end{abstract}

Keywords: structural morphology, self-organization, bionics, technology, architecture.

\section{Introduction}

Morphology is the study of configuration or the structure of animals and plants [1]. Morphology explains the shapes and arrangements of parts of organisms in terms of such general principles as evolutionary relations, function, and development [2].

The objective of structural optimization is to maximize the performance of a structure or structural component. It is driven by limited resources, environmental impact and technological competition, which demand lightweight, low cost and height-performance structures. Optimal design means the best feasible design that satisfies the prescribed performance criteria.

It is of great importance to find the best possible structural topology or layout for given design objectives and constraints at a very early stage of the design process (the conceptual and project definition phase). Thus, over the last decade, substantial and fundamental research efforts have been devoted to the 
development of efficient and reliable computational methods for structural optimization like structural shape optimization [3] and topology optimization [4] (evolutionary structural optimization methods, ESO).

These computational tools are in general not being used by architects or designers who would need simplified computational methods for form and structural optimization in the critical early stage of the design process. This paper, "Structural Morphology and Self-organization" introduces methods to bridge the gap between structural optimization theory and its practical application to structural design. It shows simplified methods and programs for form finding and topology optimization based on processes in nature.

Structural morphology is the study of form and shape of a structure and the relations between form, forces, and material, and is an important research area for structural design improvement [5]. Self-organization is a process of attraction and repulsion in which the internal organization of a system, normally an open system, increases in complexity without being guided or managed by an outside source. Self-organizing systems typically (but not always) display emergent properties [6].

The integration of structural morphology (form-finding of structure) would simplify the architectural form- and typology finding process considerably. Then structural optimization and structural morphology can also serve the process of architectural form finding. Structural morphology in this sense is to be understood as the integration of form-generating methods, structural shapeoptimizing methods and the design principles of a structure and its structural elements. In this regard, the production of component parts (CNC production), optimization in design- and production-procedures (generative design tolls for associative and parametric modelling) and assembly (automated assembly) play
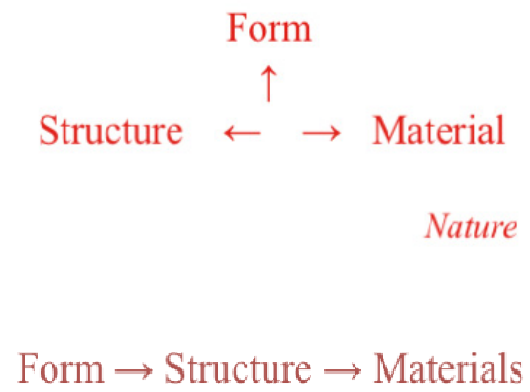

Architecture

Figure 1: In nature the form-following process, structural extension (growth) and material placement happen simultaneously and are controlled by a constant feedback loop. Architecture is most predominantly design focused and therefore the form determines the structure and the materiality. 
a crucial role. These variables can enter the morphological form-finding process as mathematical variables and constraints. In the design of free form buildings with complex geometrical structures, the goal is to define the form geometrically as early as possible. Instead of post-rationalizing double curved geometry the goal is to "pre-rationalize" the design method.

\section{What is structural optimization?}

Structural morphology occurs on three different levels: on the global scale (structural system), the local scale (structural component) and the micro scale (material). The basic ingredients for Performance-Based Optimization of Structures are the optimal 'layout', the interplay between the form-finding or morphology, the structure and the structural material. In this context the "layout" of the structure includes information on the topology, shape and sizing of the structural components and the materiality. The abstraction of the essence of optimization techniques in nature and their categorization gives an overview of general strategies for optimization processes addressing multiple problems simultaneously. It also is the starting point for simplified computational estimation strategies. The goal is not to develop a unified new optimization method, but rather to develop simplified specific methods coordinated with the structure or the form-finding process.

\subsection{Structural optimization categories}

\section{Structural Morphology: Structure Optimization + Typology Optimization + Material Optimization}

Types of structural optimization can be classified into sizing, shape and topology optimization [7].

Sizing, shape and topology optimization address different aspects of structural design problems. Typically sizing problems deal with the optimal thickness of individual members for example in a truss structure. The optimal thickness distribution in the individual member is reached in terms of peak stress or deflection etc., while equilibrium and other constraints on the state (deflection) and design variables (thickness) are satisfied. The main feature of the sizing problem is that the domain of the design model and state variables is known a priori and is fixed throughout the optimization process. In a shape optimization problem the goal is to find the optimum shape of this domain. Typology optimization finds the optimal lay-out of the structure within a specified design space. The only known quantities in this problem are the applied loads, the possible support conditions, the volume of the structure to be constructed and the additional design restrictions. In this case the physical size and the shape and connectivity of the structural elements are unknown. The topology, shape and size of the structure are not represented by standard parametric functions but by a fixed design domain (design space). Typology optimization of solid structures involves the determination of features such as the number, location and shapes of holes and the connectivity of the domain. 


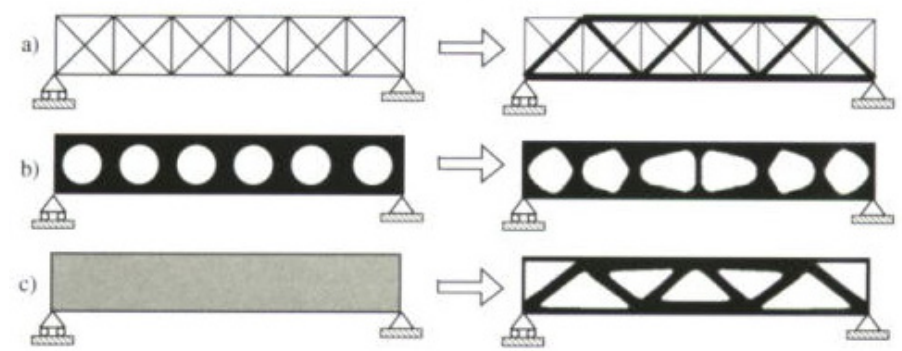

Figure 2: Three categories of structural optimization: a) sizing optimization of a truss structure; b) shape optimization; and c) topology optimization. The initial problems are shown on the left-hand side and the optimal solutions are shown on the right-hand side [8].

\section{Morphology}

The term morphology in biology refers to the outward appearance (shape, structure, colour, and pattern) of an organism and its component parts. This is in contrast to physiology, which deals primarily with function. In engineering, the term morphology could be replaced with the term structural form finding.

The term morphology was coined around the year 1800 by Johann Wolfgang von Goethe, who meant by that term a comparative doctrine of form with the goal of establishing general laws of form. Goethe's morphology is based on the assumption that every real or metaphysical being constitutes a system by its form. Form is, accordingly, the source of inward essence and of global coherence of any isolated cosmic event. Recognition of this fact leads to the doctrinal idea of form as an ontological principal of nature, i.e. as a structural principle of space.

The term morphology established itself after Goethe first in botany [9], then in zoology [10], philosophy [11] and the analysis of form [12]. In his 1948 "morphological approach", Zwicky [13] developed general morphological analysis as a method for structuring and investigating the total set of relationships contained in multi-dimensional, usually non-quantifiable, problem complexes. "Morphological analysis is simply an ordered way of looking at things" [14].

The purpose of this morphological study will be not the search for differences or fundamental contrasts, but to search for the essential principles that govern the creation of order and patterns.

In general morphology can be differentiated into the three aspects: Descriptive Morphology - The spatial relationship between elements Functional Morphology - The functional relationship between structural elements

Construction Morphology - The relationship between all structural components

Morphology as a logical concept embodies a finite aggregate of interrelated elements. This structural and thus highly typological notion allows a systematic analysis of form using mathematics and structural analysis for its description. 
The interplay between geometrical requirements and physical facts can be advantageously illustrated by the key studies shown in chapter 5 .

\subsection{On the logic of form}

Form is the result of a morphological process and may be described with the principles of the natural sciences. An examination of the term form necessarily leads to aesthetics. Because both terms are mutually dependent, it can be said that "aesthetic qualities can be analyzed." The form constructively finds its expression in structure. Structure is the logical ordering principle of all components of a construction, and can therefore be described typologically. Typology makes possible the comparative examination of structures and their states of equilibrium. In this sense, the study of form makes possible form analysis, the interpretation of the causal relationships between the form and physical forces. In the structural field, form analysis is the basis for the materialization of structures in conformity with the flow of forces. This leads to an ideal exploitation of materials and as a result to a minimization of the expenditure of materials.

In Biology morphogenesis [15] is concerned with the shapes of tissues, organs and entire organisms and the positions of the various specialized cell types. The study of morphogenesis [16] involves an attempt to understand the processes that control the organized spatial distribution of cells to form tissues, organs and overall body anatomy. Some of the earliest ideas on how physical and mathematical processes and constraints affect biological growth appear in works written by D'Arcy Wentworth Thompson [17]. These works postulate the presence of chemical signals and physio-chemical processes such as diffusion, activation and deactivation in cellular and organismic growth. D'Arcy Wentworth Thompson's central thesis 'On Growth and Form' states that the role of evolution is overemphasized, and the roles of physical laws and mechanics as determinants of the form and structure of living organisms is underemphasized. He describes the phenomenological phenomenon of living nature from a physical perspective and interprets the form of any portion of matter, [...] as due to the action of force.

This phenomenological perspective is displayed in Thompson's classic fish transformation (fig.3) in which he uses relatively simple mathematical transformations to explore the degree to which differences in the forms of related animals could be described.

In architecture structural morphology, the 'form finding of structures', deals with the study of the relation between form and structural action, and therefore includes considerations of a variety of themes such as structural behaviour, shaping structures, efficiency of structures, structural topology, flexible structures, structures in nature, innovative structures and computational morphogenesis. The research focus on the intimate relation between form and structure and material will lead to the structures of tomorrow. Typological investigations into structural configurations of spatial structures have received great attention in architecture [18] (DG Emmerich, T. Tarnai, H. Lalvani, J.K. Gabriel, R. Motro and others). 


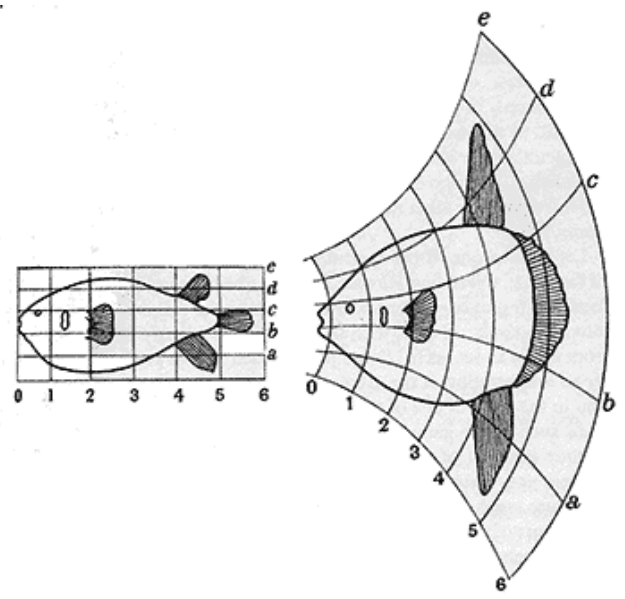

Figure 3: D'Arcy Thompson's classic fish transformation; form change through simple mathematical transformation.

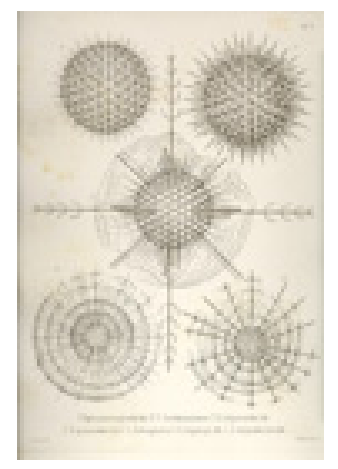

Figure 4: Planar subdivision by radial axes applied to structural morphology. The skeleton of radiolarian can be made by triangles or other types of polygons, Ernst Haeckel: Die Radiolarien (RHIZOPODA RADIARIA) Berlin, 1862.

\section{2 "Structural morphology - bridge between civil engineering and architecture"}

Structural morphology, or the 'science of form of structures,' is one of the most active research areas in architecture, aiming to bridge the gap between the fields of civil engineering and architecture, and will lead to a better understanding between the two disciplines. Structural morphology deals with the study of the relationship between the geometric form and structural behaviour. Figs. 4/5 compares the structural configuration of a natural organism with a man made structure. The geodesic dome and the ridged radiolarian shell adopt a spherical 


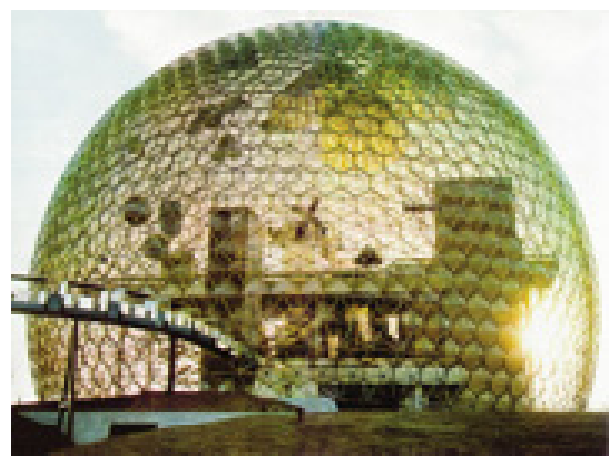

Figure 5: Geodesic dome by Buckminster Fuller.

form approximated by a polyhedron with triangular or polyhedral faces. This arrangement minimizes the amount of material in the structure as well as its weight. Both structures have no dominant bi-dimensional stress-resistant element and stresses are transmitted along the whole surface, in the case of the radiolarian as a lattice shell mash and, in Buckminster Fuller's geodesic dome, a plate shell mesh.

Structural morphology as a relatively new field of engineering is becoming more and more the centre of interest for many different and diverse disciplines. Architecture (form), structural engineering (structure), material science (new materials and properties) and mechanical engineering (thermal dynamics and building environment) are all interrelated in the form finding process. Combining structural design, morphology and materials in an integrated design approach will open up new perspectives in designing complex geometric structures [19].

Structural morphology plays an integral role in digital design and fabrication. In the last decade the increasing use of computer aided design and manufacturing has enabled the construction of buildings with complex geometries and has changed and challenged the building industry of the twenty-first century. Such projects require an integrated 3D approach with CAD, FEM (Finite Element Method), CAMP (Modelling and Prototyping) and CAB (Computer-Aided Building).

Structural morphology in architecture addresses the following key issues:

- Spatial arrangement of standing structures at static equilibrium.

- The complex relationship between force, form and material in 3D Forms: form-finding, structural morphology and optimization.

- The streamlining of interactive processes between design, engineering, analysis and manufacturing.

- The development of new materials and production methods for integrated building components.

- Engineering and prototyping of production, and the completion of construction processes.

- Influence of production methods on design / engineering components.

- Design methodology for component design and product development. 


\subsection{Smart geometry}

Architecture is fundamentally about relationships. Many of these relationships are geometric in nature or find a geometric expression. Structural engineering often relies on the ability to decompose the form into clear and independent subsystems, where the precondition to 'design' the structure is premised on the clarity of such arrangements. Contemporary architecture often escapes this and subsystems have to morph as much as the architectural form. New analysis tools are essential to avoid the severing and isolation of systems, to handle complex geometries, to harness the efficiency of interconnected subsystems, and for manufacturing.

Nature uses simple numerical rules. Shells for example use the Fibonacci numbers to generate complex geometries and structural forms and soap bubbles form bubble clusters based on the equilibrium of surface tension within the membrane.

\subsection{Self-organization}

According to the Big Bang Theory, the universe was concentrated at the beginning of time in an infinitesimally small point of infinite density and temperature. Fourteen billion years ago, this beginning state called a cosmological singularity was followed by the big bang, the explosive expansion of the universe. With the expansion of the universe, the temperature steadily declined and the synthesis of the elements began. In the course of further expansion, material fields of greater density emerged, and under the influence of gravity, galaxies, stars and planets appeared. Life in the form of informationbearing molecules, nucleic acids, appeared four billion years ago. It was no expanded, structureless, amorphous distribution of mass and energy that emerged from the original singularity of the cosmos, but rather a highly complex system built on the principles of self-organization that spans from the macro-cosmos all the way to the single atom.

The principle of self-organization is known not only in nonliving nature but also in living natural systems. The concept of self-organization plays a growing role in understanding physical analyses and supplements the Laws of Thermodynamics (condition of equilibrium). Self-organization is defined as the form-giving, shaping and limiting influences of the elements from which selforganizing systems are made. An order organized in terms of space and time emerges without outside interference.

For example, nanotubes possess a flow of energy, material and information, an independent formation of space-time conditions not brought about through outside intervention that indicates conspicuous analogs to the structural development processes of living nature. Physical characteristics play an important role in the categorization of form-finding and optimization methods, as will be shown for example with the bubble cluster. 


\subsection{Natural and technical (engineered load-bearing) structures: similarities and differences}

Engineered and natural load-bearing structures show a range of analogs and similarities. The same physical laws of mechanics underlie the structures in both areas. Form and the preservation of function are both subject to the basic principles of flow of forces and equilibrium. Construction bionics and structural bionics in particular derive knowledge for the function-, form- and load-bearing structural optimization from the analysis of natural structures (Matuschek).

Natural structures offer an abundance of observational material for structural optimization, but direct derivations in the sense of a literal imitation are not possible. The fundamental differences lie in the scale of the structures, their material composition and differences of function. Biological structures are complex in nature and cannot always be categorized into pure structural principles. Biological formation processes take place according to the laws of antagonistic principles of construction simultaneously. In ontogeny, chronologically successive and already "matured" sub-problems are solved that establish the basis and boundaries of the following stage of development. Formand functional adaptation takes place from one generation to the next.

Only through systematic abstraction will sensible knowledge for engineered construction emerge. However, the integration of form, structure and force flow management in natural formations offers an abundance of starting points for synergistic engineered structures.

Biological design principles are also functional during the building- or growth-phase. As a general rule, biological structures are subject to a mechanical feedback process, that is to say, they react adaptively to their environment. In this way, the formation as well as the structural efficiency of an organism is determined not only by the genetic code, but also by the ability of the organism in all phases of growth to establish a state of equilibrium.

\section{Structures in nature}

Self-organization is the defining principle of nature: 'Accomplishing an objective with a minimum of effort'

The translation of abstracted nature in mathematical terms and by applying prerequisite architectural considerations is the fundamental concept of form and structure analyses.

\begin{tabular}{|c|c|c|c|}
\hline NATURE & & TECHNOLOGY & \\
\hline Dynamic Adaptation & $=$ & Structure analysis & \\
\hline Evolution & $=$ & Computer Optimized Form Finding & Structural \\
\hline Genetic Code & $=$ & Digital Manufacturing & Morphogenesis \\
\hline Growth & $=$ & Construction & \\
\hline
\end{tabular}

Figure 6: Form-finding of natural structures: similarities and differences. 

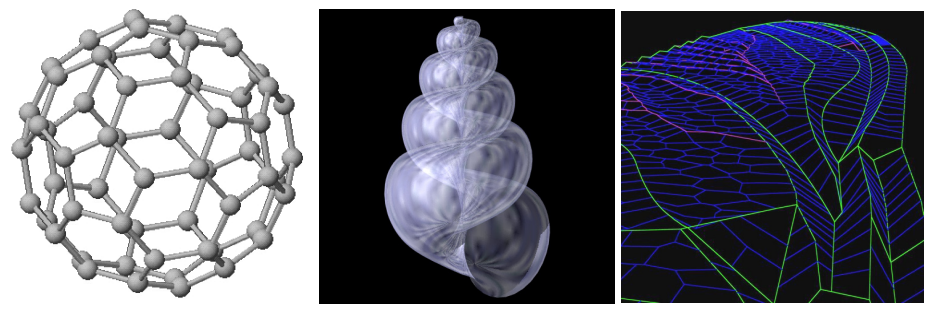

Figure 7: $\quad$ Structures in nature: buckyball carbon 40 model, sea shell computer model, dragonfly wing computer model.

Form-finding in nature is an integrated and autogenous process in the sense of self-organization. Antagonistic structural principles in nature are to be understood as form- and growth-processes that build on the equilibrium of internal and external forces or influences. In this process, growth, form, function, structure and adaptation are autogenous and self-guided. Nature avails itself of various principles in this process. The morphological blueprint and the order of processes are embedded in the object itself: as DNA in living organisms and as physical characteristics in nonliving material.

\subsection{Similarity and diversity of natural and technical structures}

\section{Structures in Nature}

- Form imbedded in system (genome, physical properties)

- Autogenous process originating or derived from sources within the same individual

- System is functioning in every stage of development

- Whole systems as well as components can grow

- Adaptable regarding external or internal changes

- System is self-monitoring

- System is growing

- System and sub-system are self-healing

- Interrelated and integral process: form, structure and materials

- Maximum efficiency and material economy

- Energy efficient, low metabolism

\section{Technical Structures}

- Separation of form, structure und material

- Structure follows form

- Economics

- Linear building process with separated process phases 


\section{Load case-adapted bio-mimetic constructing a standard technique in mechanical engineering}

This paper presents a categorization of self-organization processes in nature and abstracts these processes into simplified analytical methods.

Nature typically uses not additive, but highly integrated systems, which optimize several necessary features in one component. Energy acquired by photosynthesis or heterotrophic processes has to be diverted between growth and reproduction, and protective measures. Thereby, the efficient use of energy is critical for survival. This resulted in the evolution of strong materials and stable lightweight constructions, which present an attractive design pool for advanced technical applications.

The basis for a transfer of biological lightweight systems into technical systems (bionics/biomimetics) requires detailed studies concerning architecture and material properties of structural components in combination with the crucial functional aspects within the ecological context.

The fundament of biological constructions is the axiom of constant stress principle, which results in minimal investment and maximal performance of biogenic structures. These characteristics are also key properties for optimized technical constructions, regarding both ecological and economical aspects. It is therefore evident, that loadcase-adapted biomimetic constructing should become a standard technique in mechanical engineering.

\section{References}

[1] www.biology-online.org/dictionary/morphology

[2] www.britannica.com/EBchecked/topic/392797/morphology

[3] Bletzinger, K-U and E. Ramm, Structural optimization and form finding of lightweight structures, Computer \& Structures, Vol. 79 pp.2053-2062, 2001

[4] Cui, C., H. Ohmori and M. Sasaki, Computational Morphogenesis of 3D Structures by Extended ESO Methods, Journal of the International Association of Shell and Spatial Structures, Vol.44 n.1, pp. 51-61, 2003.

[5] Motro, R. M., Structural Morphology and Configuration Processing of Space Structures, Multi Science Publishing Co Ltd, 2009.

[6] Self Organization, Wikipedia, The Free Encyclopedia. Wikimedia Foundation.

[7] Lian, Q. Q., Performance-Based Optimization of Structures: Theory and Applications.

[8] Bendsoe, M.P. and O. Sigmund, Topology Optimization.

[9] St. Hilaires, A de, "Morphologie végétale", 1841.

[10] Haeckel, E., "Genereller Morphologie der Organismen”, 1866.

[11] Bosanquet, B., "Logic or the morphology of knowledge", 1888.

[12] Smuts, J.C. and A. Meyer-Abich ("Logik der Morphologie" 1926), H. Drieschs "Ordnungslehre", 1912, O. Spanns "Kategorienlehre", 1924, H. Friedmanns "Welt der Formen", 1926, F. Weinhandls "Gestaltanalyse", 1929. 
[13] General Morphological Analysis, 1946.

[14] Zwicky, F., "Morphological Astronomy", The Observatory, Vol. 68, No. $845,1948$.

[15] From the Greek morphê shape and genesis creation

[16] Turing, A.M., "The Chemical Basis of Morphogenesis", 1952

[17] Thompson, D.W., On Growth and Form, Dover, 1992.

[18] www.iass-structures.org and The Structural Morphology Group of IASS (Working Group No.15)

[19] Wester, T., "Structural Morphology and Natural Structures" and "Structural Morphology-A New challenge in Engineering and Architecture". Building Engineering, School of Architecture, KTH, Stockholm, 2000.

See also:

[20] Stach, E., "The morphology of sea shells" in "An Anthology of Structural Morphology" Edited by Rene 10/2009, World Scientific, Imperial College Press, pp. 172-185.

[21] Stach, E., "Synthesis of Form, Structure, and Material." On Growth and Form. Cambridge Press of Waterloo University, 5/2008.

[22] Stach, E., "Self-Generating Membrane Structures," Textile Composites and Inflatable Structures, CIMNE, Stuttgart 10/2005, pp. 153-164.

[23] Stach, E., "Form-Optimizing Processes in Biological Structures-Self generating structures in nature based on pneumatics," Textile Composites and Inflatable Structures, Springer Berlin/Heidelberg, 2005: pp. 285-304.

[24] Stach, E., "Form Finding and Optimization Method of Lightweight Membrane Structures," Textile Composites and Inflatable Structures, CIMNE, Barcelona 6/2003, pp. 218-225.

[25] Stach, E., "Smart Structures in Nature-Adaptive Lightweight Structures in Nature and Engineering," Textile composites and Inflatable Structures, CIMNE, Barcelona 6/2003, pp. 367-374.

[26] Stach, E., "Design \& Nature. Synthesis of Form, Structure and MaterialLightweight Building and New Materials," Design and Nature, WIT Press, Southampton, Boston, 9/2002, pp. 245-256. 